\title{
The acute muscular response to two distinct blood flow restriction protocols
}

\author{
SJ Dankel ${ }^{1}$, SL Buckner ${ }^{1}$, BR Counts ${ }^{1}$, MB Jessee ${ }^{1}$, JG Mouser ${ }^{1}$, KT Mattocks ${ }^{1}$, \\ GC Laurentino', T Abe $^{2}$, JP Loenneke ${ }^{1}$ \\ ${ }^{1}$ Department of Health, Exercise Science, and Recreation Management, Kevser Ermin Applied Physiology \\ Laboratory, The University of Mississippi, University, MS, USA \\ ${ }^{2}$ Department of Sport and Life Sciences, National Institute of Fitness and Sports in Kanoya, Kanoya, \\ Kagoshima, Japan
}

Received: April 10, 2016

Accepted: December 13, 2016

\begin{abstract}
The purpose of this study was to determine acute physiological and perceptual responses to two commonly implemented blood flow restriction protocols. Using a within-subject design, 15 participants (age 25) performed four sets of unilateral elbow flexion with each arm. One arm exercised using a $3-\mathrm{cm}$ elastic cuff inflated to $160 \mathrm{mmHg}$, whereas the other arm exercised using a $5-\mathrm{cm}$ nylon cuff inflated to $40 \%$ of the individual's arterial occlusion pressure. While both protocols elicited increases in acute muscle thickness [pre: $4.5(0.2) \mathrm{cm}$, post: $5.0(0.2) \mathrm{cm} ; p<0.001$ ] and electromyography amplitude [first 3 reps: 55 (5) \%MVC; last 3 reps: 87 (10) \%MVC], there were no differences between conditions. Both protocols produced decreases in post-exercise strength (pre: $70 \mathrm{Nm}$, post: $51 \mathrm{Nm} ; p<0.001)$ with no difference between conditions. The nylon protocol resulted in more repetitions during sets $2[13$ (2) vs. $9(4) ; p=0.001]$ and $3[10$ (2) vs. $7(4) ; p=0.05]$, while producing lower levels of discomfort following each set (average 3 vs. $4 ; p<0.05$ ). In conclusion, both protocols produced similar acute responses thought to be important for promoting muscle growth. However, the use of arbitrary pressures may place some individuals under complete arterial occlusion which may increase the potential risk of an adverse event.

Keywords: elbow flexion, KAATSU, occlusion training, resistance training, strength
\end{abstract}

\section{Introduction}

The American College of Sports Medicine recommends that individuals train with at least $70 \%$ of their one-repetition maximum (1RM) to provide a sufficient stimulus capable of inducing muscle hypertrophy (1). It has since been shown that the external load lifted seems to be of less importance provided a sufficient stimulus is applied (20). One mode of training, termed blood flow restriction (BFR) training, involves placing a pneumatic cuff or elastic wrap at the most proximal part of the arms or legs. The goal of BFR is to limit arterial blood flow into the muscle while also occluding venous return, and has been shown to decrease the number of repetitions necessary to elicit muscle growth (12). Therefore, BFR in combination with low-load resistance training has been shown to increase muscle size and strength with loads as low as $20 \%(10)$ or $30 \%(5,23,26,30-32) 1 \mathrm{RM}$, using protocols that otherwise would not result in muscle hypertrophy.

Corresponding author: Jeremy Paul Loenneke, $\mathrm{PhD}$

Department of Health, Exercise Science, and Recreation Management, Kevser Ermin Applied Physiology Laboratory, The University of Mississippi

P.O. Box 1848, University, MS 38677, USA

Phone: +1 662915 5567; Fax: +1 662915 5525; E-mail: jploenne@olemiss.edu 
While the practical application of BFR with elastic wraps has been demonstrated to be effective $(18,19,28)$ and may be more easily implemented, the use of pneumatic cuffs allows for the regulation of pressures that are applied. The two different pneumatic cuff types commonly used involve either an elastic (inflated by the KAATSU Master Apparatus, Tokyo, Japan) or nylon (inflated by the Hokanson Inc., Bellevue, WA, USA) cuff (8). When implemented with upper-body exercise, the elastic cuff is often inflated to an arbitrary pressure (i.e., the same pressure for all individuals) of $160 \mathrm{mmHg}$ for the duration of exercise $(23,30-32)$, whereas the nylon cuff is often inflated to a percentage of the individual's arterial occlusion pressure $(2,5,9-11)$. While both protocols would appear to effectively increase muscle size, no previous research has examined the percentage of relative arterial occlusion pressure applied with the elastic cuff inflated to an arbitrary $160 \mathrm{mmHg}$. It has been suggested that the use of arbitrary pressures may result in some individuals being administered pressures that are too low and inadequate for inducing muscle growth, whereas other individuals may be prescribed higher pressures that result in complete arterial occlusion, thus hypothetically increasing the likelihood of an adverse event (15).

Despite two distinct protocols used for the implementation of BFR (6), no study to date has examined the differential effects of these differing protocols as it relates to acute changes in muscle isometric strength [maximal voluntary contraction (MVC)], muscle thickness, and electromyography (EMG) amplitude. As such, the comparison of these protocols is of great interest given the heterogeneity and difficulty to generalize such wide spread differences which involve differences in cuff sizes, cuff materials, and restrictive pressures (6). The acute measures of muscle thickness and EMG amplitude may provide insight relating to chronic changes in muscle size as both acute muscle thickness (i.e., swelling) (13) and muscle activation (29) have been proposed as important mechanisms responsible for the increased muscle size resulting from low-load resistance training with BFR. Additionally, post-exercise muscle strength measures may provide information on the fatigue response from exercise, and while perceptual responses to these differing cuff materials do not appear different in the lower body (16), they have not been addressed in the upper body and may differ based on the large differences in limb size or protocols employed.

Therefore, the purpose of this study was to determine the differential effects of using two distinct, and commonly used, protocols for the implementation of BFR. It was hypothesized that there would be no changes in RPE; however, discomfort would be higher from exercise with the elastic cuff given the estimated greater relative pressure. Additionally, we hypothesized no differences would be observed in relation to EMG amplitude, muscle thickness, or post-exercise isometric strength between arms due to both protocols bringing participants to, or close to, volitional fatigue by the fourth set.

\section{Materials and Methods}

\section{Subjects}

A total of 17 young resistance trained (regularly performing upper-body resistance training) males and females volunteered to participate in the study. Two individuals did not complete all of the testing sessions; therefore, 15 individuals ( 12 males, 3 females) were included in the final analyses. For the first visit, all participants were instructed to refrain from: (a) eating $2 \mathrm{~h}$ prior and (b) consuming caffeine $8 \mathrm{~h}$ prior. In addition, participants were instructed to refrain from exercise for $24 \mathrm{~h}$ prior to each visit. On the first visit, participants entered the laboratory 
and filled out an informed consent form, adult health history questionnaire, and physical activity readiness questionnaire. Participants were excluded from the study if they were not between the ages of 18 and 35, were smokers, had an orthopedic injury preventing exercise, or had one or more predisposing risk factors for thromboembolism (22). The specific risk factors for thromboembolism included having a body mass index $\geq 30$, diagnosis of Crohn's disease, past fracture of the hip pelvis or femur, major surgery within 6 months, diagnosis of varicose veins, or a past personal or family history of deep vein thrombosis or pulmonary embolism. The study was approved by the University's Institutional Review Board and all participants provided informed written consent prior to participation.

\section{Study design}

Participants visited the laboratory at the same time of day on two separate occasions separated by a 3-week period [this study was part of a greater project assessing methodological differences between cuffs (4)]. The participants' strength level was not expected to change appreciably over the course of 3 weeks and the pre-exercise MVC values across the 3 weeks confirmed this (data not shown). On day 1, participants were randomly assigned to have one arm train using a 3-cm-wide elastic cuff (KAATSU Master Apparatus) and the other arm using a 5-cm-wide nylon cuff (Hokanson Inc.). Individuals then had their arterial occlusion pressure measured using the specific cuff they would be exercising with on visit 2. Following the arterial occlusion measurements, 1RM strength of the elbow flexors and familiarization with the MVC testing procedures were completed for both arms in a randomized fashion. Three weeks later, participants visited the laboratory a second time to complete the elbow flexion exercise on both arms in a counterbalanced fashion with the appropriate training device and cuff. Participants were first measured for muscle thickness, then proceeded to complete a baseline MVC that we used for normalization of our EMG data, and to allow for a baseline strength measure to assess fatigue. Ratings of perceived exertion (RPE) and discomfort were also obtained before exercise. Individuals then performed four sets of low-load BFR exercise on each arm while EMG amplitude, RPE, and discomfort were obtained throughout exercise as detailed below. At the completion of all four sets, MVC and muscle thickness measurements were taken immediately after exercise, as well as 5-, 20-, 40-, and 60-min post-exercise. The same procedures were then completed on the opposite arm using the other pneumatic cuff and inflation device.

\section{Arterial occlusion}

The standing arterial occlusion pressure was determined as the minimal pressure in which a pulse was no longer present at the radial artery using an MD6 Doppler Probe (Hokanson Inc.). For use with the E20 rapid cuff inflator, the pressure was inflated to $50 \mathrm{mmHg}$ before being progressively increased by $1 \mathrm{mmHg}$ increments until a pulse was no longer detected. For the elastic cuff (inflated by the KAATSU Master Apparatus), the pressure cuff was applied to an initial pressure of $30 \mathrm{mmHg}$ (i.e., the pressure applied to the arm prior to the inflation of the cuff) and then inflated to a pressure of $50 \mathrm{mmHg}$. The pressure was increased in approximate $50 \mathrm{mmHg}$ increments before being slowly adjusted by $10 \mathrm{mmHg}$ increments until the identification of the lowest pressure in which a pulse was no longer present. The elastic cuff was increased or decreased by $10 \mathrm{mmHg}$ to find the arterial occlusion pressure because the machine regulating the elastic cuff pressure (KAATSU Master Apparatus) cannot be adjusted in smaller increments. 
$1 R M$

A 1RM for the unilateral elbow flexor exercise was obtained on both arms for each individual on day 1. Briefly, participants warmed up with a relatively low weight corresponding to an estimated 30\% 1RM. Following the brief warm-up, the load was increased to approximately $90 \%$ of the individuals $1 \mathrm{RM}$ and participants performed one repetition. Thereafter, the load was adjusted to an estimated 1RM and the load was either increased or decreased in $0.5 \mathrm{~kg}$ increments until a $1 \mathrm{RM}$ was obtained. The dumbbell was handed to each individual at full elbow extension and participants were instructed to maintain their back and heels against the wall during all 1RM attempts to ensure strict form. Each attempt was separated by about $2 \mathrm{~min}$ of rest and the individual performed as many attempts as necessary until we determined the maximum load in which they could perform (i.e., they could not complete a repetition with the next smallest incremental increase in load). A 1RM was usually obtained between 3 and 5 repetitions. Only repetitions that were completed with proper form were counted.

\section{BFR protocols}

The two distinct protocols both included inflating a pressure cuff at the most proximal part of the arm before exercise and remained inflated until completion of the final set. The two protocols were as follows: (a) a 3-cm-wide elastic cuff (KAATSU Master Apparatus) with an initial pressure of $30 \mathrm{mmHg}$ was applied before using the KAATSU device to inflate the cuff to an arbitrary pressure of $160 \mathrm{mmHg}$ and (b) a 5-cm-wide nylon cuff (Hokanson Inc.) was inflated by the E20 rapid cuff inflator to $40 \%$ of the individuals pre-determined resting arterial occlusion pressure. The arbitrary pressure of $160 \mathrm{mmHg}$ was chosen for the elastic cuff as this exact pairing of cuff size, cuff material, and cuff pressure has been used previously throughout the BFR literature $(23,30-32)$, and $40 \%$ arterial occlusion was chosen for the nylon cuff as this is the lowest relative pressure which has been shown to provide beneficial muscle adaptations $(5,11)$.

\section{EMG amplitude}

EMG amplitude was recorded from the biceps brachii of the arm during exercise. Electrodes were placed on a line between the medial acromion and the antecubital fossa at a distance of $1 / 3$ from the antecubital fossa. The skin was shaved, abraded, and cleaned with alcohol wipes. Bipolar electrodes were placed over the muscle belly with an inter-electrode distance of $20 \mathrm{~mm}$ and the ground electrode was placed on the 7th cervical vertebrae at the neck (7). These Surface EMG for Non-Invasive Assessment of Muscles (SENIAM) procedures were used to ensure the electrodes were placed on the same location for each of the two conditions. The surface electrodes were connected to an amplifier and digitized (iWorx, Dover, NH, USA). The signal was filtered (low-pass filter $500 \mathrm{~Hz}$; high-pass filter $10 \mathrm{~Hz}$ ), amplified $(1,000 \times)$, and sampled at a rate of $1 \mathrm{kHz}$. Before the exercise bout, the participants performed 2 isometric MVCs with the biceps brachii at a joint angle of $60^{\circ}$ with 60 -s rest between MVCs on an isokinetic dynamometer. EMG amplitude was recorded continuously from the biceps brachii during each exercise bout. A computer software program (iWorx) was used to analyze the data. EMG amplitude (root mean square) was analyzed from the average of the first 3 repetitions and the average of the last 3 repetitions for each set and expressed relative to the highest pre-exercise MVC (\%MVC). 


\section{Elbow flexion exercise}

All participants performed four sets of unilateral elbow flexion exercise with both arms using a load corresponding to $30 \%$ of their predetermined $1 \mathrm{RM}$ for each respective arm. The exercise consisted of one set of 30 repetitions followed by three sets of 15 repetitions, with 30 -s rest allotted between the sets. Participants lifted to the beat of a metronome allowing $1 \mathrm{~s}$ for each the concentric and eccentric portion of the exercise. If participants were unable to lift the weight through the full range of motion with proper form, or could not maintain the beat of the metronome, they were stopped. The same protocol was performed on both arms separated by $15 \mathrm{~min}$ of rest.

\section{RPE and discomfort}

Participants were explained in depth how to rate their RPE and discomfort to ensure they understood the scale being used. Immediately before and after each set of exercise individuals were asked to rate their level of exertion using the standard Borg 6-20 scale (3). A rating of discomfort was obtained using Borg's Discomfort Scale (CR10+) as described previously (13). Participants were explained that the scale was rated from 0 to 10 with a score of 10 representing their previously worst felt discomfort. They were then instructed that a rating of 10 was their reference point and they could exceed 10 if the discomfort they felt was greater than what they have ever felt before. Participants were then asked if they had any questions. All participants fully understood the scale prior to exercise. The ratings of discomfort were taken immediately before exercise, as well as $20 \mathrm{~s}$ after sets 1, 2, and 3, and immediately after set 4 . Discomfort was taken $20 \mathrm{~s}$ after each set because participants in previous studies anecdotally noted greater discomfort later in the rest periods and we felt this provided a more accurate representation of how discomforting the protocol was.

\section{$M V C$}

Participants were asked to sit in an isokinetic dynamometer (Biodex System 4 Medical Systems, Shirley, NY, USA), with the seat and lever arm adjusted for each individual. After weighing the limb to correct for gravity, the lever arm locked into place at $60^{\circ}$ of elbow flexion. Participants were then asked to contract their bicep by pulling against the lever arm as hard as possible for a 3 -s period. Participants performed 2 contractions separated by a 1-min rest period on each arm, with the exception of the initial post-exercise MVC in which only one contraction was completed to avoid allowing additional recovery time. The maximum amount of torque that individuals produced was recorded as their MVC for that particular time point. Participants were blinded from their MVC results during testing.

\section{Muscle thickness}

The length of each arm was measured and the site for muscle thickness was measured at $70 \%$ of the distance between the acromion process and lateral epicondyle. An Aloka SSD-500 B-mode ultrasound (Aloka Co. Ltd., Tokyo, Japan) was used to measure the distance between the musclefat and muscle-bone interface. The image was obtained by placing a probe on the individual while using conductive gel to avoid depressing the skin. Images were then freeze framed and measured twice at each time point and the average of the two measurements was recorded.

\section{Statistical analysis}

To determine differences in cuff pressures applied during rest and exercise, a paired sample $t$-test was used. A $2 \times 6$ (condition $\times$ time) repeated measures analysis of variance (ANOVA) 
was used to compare changes in acute muscle thickness and isometric torque between cuff types at pre, post, 5, 20, 40, and 60-min post-exercise and a $2 \times 4$ repeated measures ANOVA was used to determine differences in EMG amplitude and repetitions completed between different cuffs across each of the four sets. If there was an interaction, a paired sample $t$-test was used to determine differences between cuffs within each time point and a one-way repeated measures ANOVA was used to identify differences across time within each cuff. If there was no interaction, then main effects were interpreted. To determine differences in the perceptual response (RPE and discomfort) between cuffs within each set a non-parametric Wilcoxon paired $t$-test was employed. Tests were not done across each set as we were only interested in investigating perceptual differences between cuffs, not across time within each cuff. Significance for all tests was set at $p<0.05$. All analyses were performed using SPSS version 23 (IBM, Armonk, NY, USA).

\section{Results}

All results are expressed as mean (standard deviation), with the exception of RPE and discomfort which are expressed as median (25th percentile and 75th percentile). All of the data met the assumptions of the employed tests (i.e., normality and sphericity).

\section{Demographics}

A total of 15 resistance trained males $(n=12)$ and females $(n=3)$ completed the study protocol. Information on the study population is as follows: age - 25 (2) years; height - 199 (11) cm; body mass index - 82 (11) kg; arm circumference - 34.8 (4) cm; $1 \mathrm{RM}-23.5(7.8) \mathrm{kg}$.

\section{Restrictive pressure}

The arterial occlusion pressure (i.e., pressure required to cutoff blood flow) was greater in the elastic cuff when compared with the nylon cuff [262 (64) vs. 139 (13) $\mathrm{mmHg} ; p<0.001]$. The $40 \%$ arterial occlusion in the nylon cuff required participants to exercise at a lower inflation pressure of 57 (6) $\mathrm{mmHg}$ in comparison to the arbitrary $160(0) \mathrm{mmHg}$ pressures used for the elastic cuff during exercise $(p<0.001)$. In terms of the relative pressure applied (applied pressure/arterial occlusion pressure), the elastic cuff produced a greater relative occlusion pressure that averaged $65(19) \%$ and was highly variable $(\min =43 \%$, $\max =$ $106 \%$ ) in comparison to the constant $40(0) \%$ relative occlusion pressure used during the nylon cuff protocol.

\section{Repetitions}

There was a condition $\times$ time interaction for repetitions $(p=0.038)$ with the nylon cuff resulting in a greater number of repetitions during sets $2(p=0.001)$ and $3(p=0.05)$ when compared with the elastic cuff (Fig. 1). Both protocols produced significant reductions in repetitions across sets: with the nylon cuff protocol: set $1>\operatorname{set} 2>\operatorname{set} 3>\operatorname{set} 4$ and the elastic cuff protocol: set $1>$ set $2>$ set $3=\operatorname{set} 4$ (Fig. 1).

\section{Acute muscle thickness}

There was no interaction $(p=0.31)$ for acute muscle thickness, so main effects were analyzed. While there was no main effect of condition $(p=0.689)$, there was a main effect 


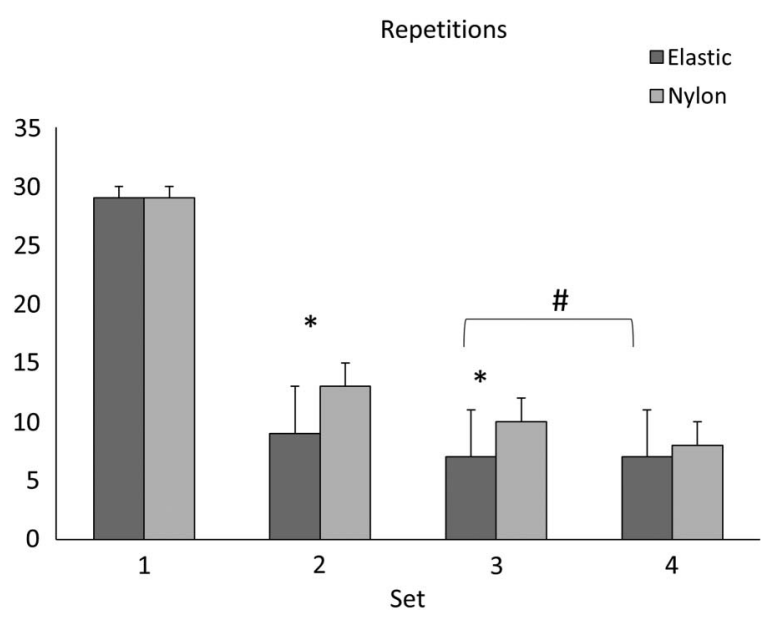

Fig. 1. A significant interaction was present with the nylon cuff resulting in a significantly greater number of repetitions during sets 2 and 3. Notably, repetitions decreased across sets for both protocols with the exception of sets 3 and 4 in the elastic cuff. *Significantly different between protocols. " Not statistically significant across time
A

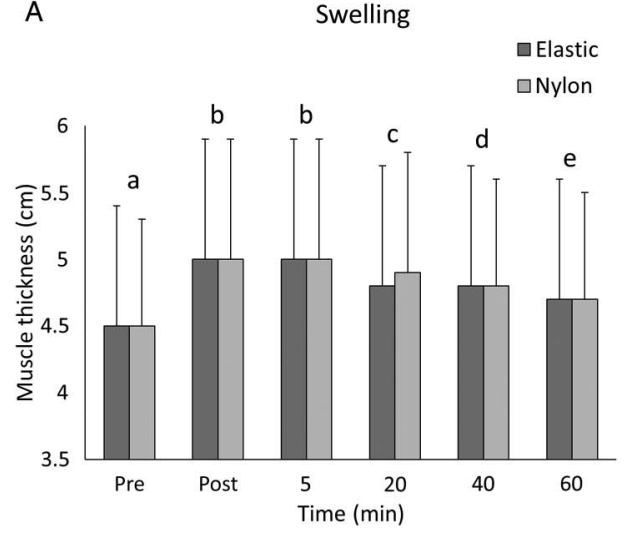

B
Torque

$\square$ Elastic

$\square$ Nylon

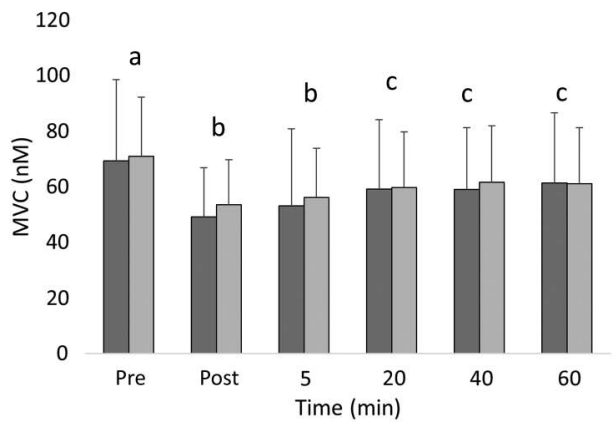

Fig. 2. Acute responses measured before and up to 60-min post-exercise. (A) A main effect of time was present for acute muscle thickness (swelling). Different letters represent time points being significantly different from one another. (B) A main effect of time was present for torque. Different letters represent time points being significantly different from one another

of time $(p<0.001)$. Acute muscle thickness appeared to increase and remain elevated 5-min post-exercise before gradually declining over the 60 -min post-exercise period (Fig. 2A).

\section{Maximal voluntary isometric strength}

There was no interaction $(p=0.31)$ for torque, so main effects were analyzed. While there was no main effect of condition $(p=0.37)$, there was a main effect of time $(p<0.001)$ with lower torque produced post-exercise. Strength recovery from exercise began at the 20 -min post-exercise time point but was still suppressed at the 60 -min post-exercise time point when compared with baseline values (Fig. 2B).

\section{EMG amplitude}

There was no interaction for EMG amplitude following the first $3(p=0.187)$ or last 3 repetitions $(p=0.40)$ of each set, so main effects were interpreted. During the first 3 
Table I. Surface electromyography (EMG) of the biceps during the first 3 and last 3 repetitions of each set

\begin{tabular}{|l|c|c|}
\hline \multicolumn{3}{|c|}{ EMG amplitude (\%MVC) } \\
\hline \multicolumn{3}{|c|}{ Elastic } \\
\hline First 3 reps & $55(24)$ & $55(24)$ \\
\hline Set 1 & $74(34)$ & $79(46)^{*}$ \\
\hline Set 2 & $71(34)$ & $83(47)^{*}$ \\
\hline Set 3 & $77(41)$ & $84(51)^{*}$ \\
\hline Set 4 & & $94(48)$ \\
\hline Last 3 reps & $85(33)$ & $106(66)^{\#}$ \\
\hline Set 1 & $88(39)$ & $92(49)$ \\
\hline Set 2 & $82(35)$ & $92(56)$ \\
\hline Set 3 & $82(41)$ & \\
\hline Set 4 & & \\
\hline
\end{tabular}

*Both protocols significantly different from set 1 .

${ }^{\#}$ Both protocols significantly different from sets 3 and 4

repetitions, there was no main effect of condition $(p=0.58)$, however, there was a main effect of time $(p<0.001)$ demonstrating lower EMG amplitude in set 1 compared with all other sets (Table I). Additionally, during the last 3 repetitions, there was no main effect of condition $(p=0.39)$, however, there was a main effect of time with greater EMG amplitude in set 2 compared with sets $3(p=0.01)$ and $4(p<0.001)$ (Table I).

\section{$R P E$}

There were no differences in RPE at rest $(p=0.99)$. While no differences were observed in RPE during sets 2, 3, and 4, the RPE during set 1 was significantly greater during exercise with the elastic cuff in comparison to the nylon cuff (Fig. 3A; $p=0.03$ ).

\section{Discomfort}

There were no differences in discomfort between protocols at rest $(p=0.31)$, however, the elastic cuff resulted in a greater discomfort following each set in relation to the nylon cuff [set $1(p=0.01)$, set $2(p=0.002)$, set $3(p=0.002)$, and set $4(p=0.003)$ ] (Fig. 3B).

\section{Discussion}

This is the first study to compare upper-body BFR exercise implemented with a nylon cuff inflated to a relative pressure of $40 \%$ of the individual's arterial occlusion, with that of another commonly used protocol involving an elastic cuff inflated to an arbitrary absolute pressure of $160 \mathrm{mmHg}$ for all individuals. Despite differences in applied pressures, repetitions and perceptual responses, the measures of EMG amplitude, acute muscle thickness, and postexercise MVC strength did not differ between protocols. 

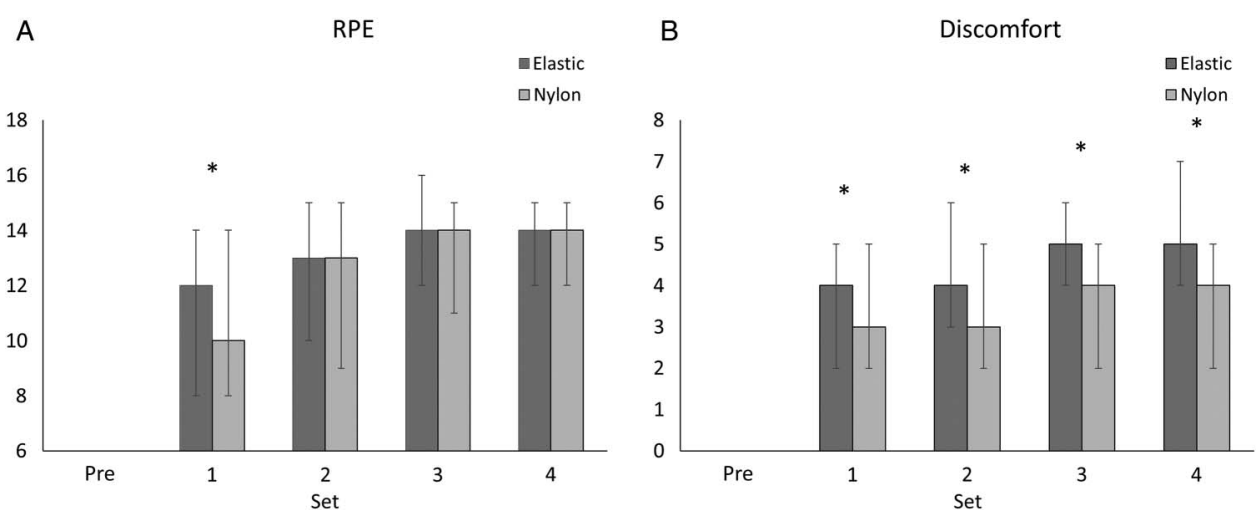

Fig. 3. (A) Illustration of perceptual responses to each exercise protocol. Ratings of perceived exertion (RPE) across sets for both protocols. (B) Discomfort across sets for both protocols. *Significantly different between protocols

While elastic and nylon cuffs have been compared with the lower body (16), the previously conducted experiment compared both cuffs inflated to the same relative pressures, which does not give an accurate comparison of the actual protocols used. Therefore, differing from this previous study, we did not use similar relative pressures but rather used differing pressures comparing a relative pressure of $40(0) \%$ arterial occlusion using a nylon cuff with an absolute arbitrary pressure of $160 \mathrm{mmHg}$ using the elastic cuff. Notably, this arbitrary pressure corresponded to an average relative pressure of 65 (19)\% and this pressure difference may partially explain the discrepancy in repetitions (Fig. 1), RPE (Fig. 3A) and discomfort (Fig. 3B) found in this study were not observed in the lower body (16). It can be reasoned that the higher pressures used in the elastic cuff in this study may have resulted in the higher RPE and discomfort scores given (14). It should be mentioned, however, that $65 \%$ was simply the average arterial occlusion pressure and varied greatly across individuals $(\min =43 \%$, $\max =106 \%)$.

The similar acute responses observed in this study support previous research demonstrating that both of the examined protocols effectively promote long-term muscle adaptations to BFR exercise $(5,11,23,30-32)$. Notably, the similar increases in EMG amplitude, particularly during the final 3 repetitions (Table I), are of great importance as muscle hypertrophy is thought to be dependent on high levels of muscle activation (21). These similarities in EMG amplitude despite volume and pressure differences have been reported previously comparing different relative pressures (5). Additionally, muscle swelling has been thought to be a mechanism responsible for muscle growth (13), and both protocols elicited similar increases in acute muscle thickness (Fig. 2A). Finally, the similar decline in MVC torque post-exercise demonstrates that both protocols likely induced a similar level of fatigue (Fig. 2B). These similarities between protocols, despite the differences in pressures applied, support previous research illustrating similar chronic muscle adaptations following training under $40 \%$ and $90 \%$ arterial occlusion pressures (5). Therefore, it would seem to reason that a wide range of pressures can elicit similar muscle adaptations, and given the higher ratings of discomfort present in the elastic cuff group exercising under a higher pressure, performing BFR whereas using lower relative pressures may be a more palatable stimulus capable of reducing participant discomfort. 
Although both protocols would appear equally effective for inducing short-term and long-term muscle adaptations, a major difference in these protocols lies in the individualization and standardization of the applied stimulus. This was evident in that the $160 \mathrm{mmHg}$ arbitrary pressure applied in conjunction with the elastic cuff protocol produced a wide range of relative pressures ranging from as low as $43 \%$ to as high as $106 \%$ of the individual's arterial occlusion pressure. Furthermore, one individual was excluded from the analysis of relative pressures given the device used to inflate the elastic cuff only goes up to $500 \mathrm{mmHg}$ and this did not occlude blood flow in this individual. Therefore, one individual was exercising at a pressure lower than $40 \%$ arterial occlusion, although the exact relative pressure used remains unknown.

The individual differences in arterial occlusion pressures illustrate that while some individuals were placed under complete arterial occlusion, others were prescribed relatively low restrictive pressures, making it difficult to compare these results among individuals, and impossible to replicate across studies. Additionally, while BFR would appear to be a safe stimulus (17), any adverse event occurring during BFR exercise would likely occur under higher pressures (15). Therefore, applying a relative pressure eliminates the risk of applying too low of a pressure incapable of inducing hypertrophy, or, although it has never been observed, applying too high of a pressure increasing the potential risk of an adverse event during exercise. Previous studies suggesting that BFR training may impose greater cardiovascular risks $(24,27)$ have failed to consider the importance of applying appropriate relative pressures, as greater levels of restriction evoke greater cardiovascular responses (25). Also, making the applied pressure relative to the individual would eliminate the risk of completely occluding arterial blood flow during exercise.

In addition to making the stimulus relative to the individual, the large pressure difference required to reach arterial occlusion illustrates the importance of also making the BFR stimulus relative to the cuff being used. For example, the arbitrary $160 \mathrm{mmHg}$ used for all individuals with the elastic cuff would have placed most individuals under complete arterial occlusion using the nylon cuff, whereas this pressure only corresponded to about $65 \%$ of the arterial occlusion pressure when used with the elastic cuff. This discrepancy may have been due to the slightly smaller width of the elastic $(3 \mathrm{~cm})$ in comparison to the nylon cuff $(5 \mathrm{~cm})$, however, it seems unlikely this small difference would have such a large impact on the arterial occlusion measurement. It can be hypothesized that the smaller limbs of the upper body may have reduced some of the "rebounding" effect of the elastic cuff compressing against the vasculature, given the elastic cuff applies a measurable initial pressure (i.e., the pressure applied by applying the cuff before inflation). An alternate explanation may be that the initial pressure applied in this study was $30 \mathrm{mmHg}$ as we replicated what is used in combination with this protocol $(23,30-32)$, whereas the previous study examining cuff differences in the lower body implemented an initial pressure of $50 \mathrm{mmHg}$ (16). Therefore, similar to what was previously observed in the lower body (16), applying an initial pressure of $50 \mathrm{mmHg}$ in the upper body may have produced a restrictive pressure more commonly representative of what is applied with the nylon cuff, although the initial pressure cannot be quantified when using the nylon cuff. Although speculative, this would detail the importance of reporting the initial pressure applied when using the elastic cuff.

\section{Limitations}

As with all studies, this study is not without limitations. First off, this study design sought to compare two commonly used BFR protocols, and therefore we cannot decipher specifically 
between cuff materials, protocols, or inflation devices. Additionally, the devices we used to inflate each of the pressure cuffs were different, and although we tried to use the same device to inflate each cuff, we were unsuccessful in doing so. Despite this potential limitation, the purpose of this study was to take a practical approach on comparing protocols that are commonly used within the BFR literature, and these commonly used protocols often employ the inflation devices used in this study. Additionally, we cannot rule out the possibility that the individual's arterial occlusion pressure may have changed slightly over the 3-week period between the arterial occlusion measurement and exercise session, but this would seem unlikely to have a measurable effect on the results observed.

\section{Conclusion}

In conclusion, our results demonstrate that EMG amplitude, acute muscle thickness (i.e., swelling), and post-exercise muscle strength (i.e., fatigue) do not differ when comparing upper-body BFR exercise with elastic cuffs inflated to arbitrary pressures and nylon cuffs inflated to relative pressures. While both arbitrary and relative pressures appear to produce similar acute responses, this study may provide some insight into the potential issues with using arbitrary pressures given the lack of individualization and hypothetically greater safety risks likely involved with exercising under complete arterial occlusion. Future studies may want to avoid the simplistic approach of using one set arbitrary pressure for all individuals, and seek to implement the use of relative pressures to consider for individual differences as well as differences in the restriction apparatus used.

\section{Acknowledgements}

This study was not supported by any external funding. The authors are not aware of any affiliations, memberships, funding, or financial holdings that might be perceived as affecting the objectivity of this manuscript.

\section{Conflict of interest}

None declared.

\section{REFERENCES}

1. American College of Sports Medicine: American College of Sports Medicine position stand. Progression models in resistance training for healthy adults. Med. Sci. Sports Exerc. 41(3), 687-708 (2009)

2. Barnett BE, Dankel SJ, Counts BR, Nooe AL, Abe T, Loenneke JP: Blood flow occlusion pressure at rest and immediately after a bout of low load exercise. Clin. Physiol. Funct. Imaging 36(6), 436-440 (2015)

3. Borg GA: Psychophysical bases of perceived exertion. Med. Sci. Sports Exerc. 14(5), 377-381 (1982)

4. Buckner SL, Dankel SJ, Counts BR, Jessee MB, Mouser JG, Mattocks KT, Laurentino GC, Abe T, Loenneke JP: Influence of cuff material on blood flow restriction stimulus in the upper body. J. Physiol. Sci. 67, 207-215 (2017)

5. Counts BR, Dankel SJ, Barnett BE, Kim D, Mouser JG, Allen KM, Thiebaud RS, Abe T, Bemben MG, Loenneke JP: The influence of relative blood flow restriction pressure on muscle activation and muscle adaptation. Muscle Nerve 53(3), 438-445 (2015)

6. Dankel SJ, Jessee MB, Abe T, Loenneke JP: The effects of blood flow restriction on upper-body musculature located distal and proximal to applied pressure. Sports Med. 46(1), 23-33 (2016)

7. Hermens HJ, Freriks B, Disselhorst-Klug C, Rau G: Development of recommendations for SEMG sensors and sensor placement procedures. J. Electromyogr. Kinesiol. 10(5), 361-374 (2000) 
8. Ingram J, Loenneke JP: The current state of blood flow restriction 2015. Available at: https://www.dropbox.com/ s/qmkxwzncpjrr0q2/OleMiss_BloodFlowRestriction.pdf?dl=0. Accessed 25 November 2015

9. Laurentino GC, Loenneke JP, Teixeira EL, Nakajima E, Iared W, Tricoli V: The effect of cuff width on muscle adaptations after blood flow restriction training. Med. Sci. Sports Exerc. 48(5), 920-925 (2015)

10. Laurentino GC, Ugrinowitsch C, Roschel H, Aoki MS, Soares AG, Neves M Jr, Aihara AY, Fernandes Ada R, Tricoli V: Strength training with blood flow restriction diminishes myostatin gene expression. Med. Sci. Sports Exerc. 44(3), 406-412 (2012)

11. Lixandrão ME, Ugrinowitsch C, Laurentino G, Libardi CA, Aihara AY, Cardoso FN, Tricoli V, Roschel H: Effects of exercise intensity and occlusion pressure after 12 weeks of resistance training with blood-flow restriction. Eur. J. Appl. Physiol. 115, 2471-2480 (2015)

12. Loenneke JP, Balapur A, Thrower AD, Barnes J, Pujol TJ: Blood flow restriction reduces time to muscular failure. Eur. J. Sport Sci. 12(3), 238-243 (2012)

13. Loenneke JP, Fahs CA, Thiebaud RS, Rossow LM, Abe T, Ye X, Kim D, Bemben MG: The acute muscle swelling effects of blood flow restriction. Acta Physiol. Hung. 99(4), 400-410 (2012)

14. Loenneke JP, Kim D, Fahs CA, Thiebaud RS, Abe T, Larson RD, Bemben DA, Bemben MG: The effects of resistance exercise with and without different degrees of blood-flow restriction on perceptual responses. J. Sports Sci. 33, 1472-1479 (2015)

15. Loenneke JP, Thiebaud RS, Abe T, Bemben MG: Blood flow restriction pressure recommendations: the hormesis hypothesis. Med. Hypotheses 82(5), 623-626 (2014)

16. Loenneke JP, Thiebaud RS, Fahs CA, Rossow LM, Abe T, Bemben MG: Blood flow restriction: effects of cuff type on fatigue and perceptual responses to resistance exercise. Acta Physiol. Hung. 101(2), 158-166 (2014)

17. Loenneke JP, Wilson JM, Wilson GJ, Pujol TJ, Bemben MG: Potential safety issues with blood flow restriction training. Scand. J. Med. Sci. Sports 21(4), 510-518 (2011)

18. Lowery RP, Joy JM, Loenneke JP, de Souza EO, Machado M, Dudeck JE, Wilson JM: Practical blood flow restriction training increases muscle hypertrophy during a periodized resistance training programme. Clin. Physiol. Funct. Imaging 34(4), 317-321 (2014)

19. Luebbers PE, Fry AC, Kriley LM, Butler MS: The effects of a 7-week practical blood flow restriction program on well-trained collegiate athletes. J. Strength Cond. Res. 28(8), 2270-2280 (2014)

20. Mitchell CJ, Churchward-Venne TA, West DDW, Burd NA, Breen L, Baker SK, Phillips SM: Resistance exercise load does not determine training-mediated hypertrophic gains in young men. J. Appl. Physiol. 113(1), 71-77 (2012)

21. Morton RW, McGlory C, Phillips SM: Nutritional interventions to augment resistance training-induced skeletal muscle hypertrophy. Front. Physiol. 6, 245 (2015)

22. Motykie GD, Zebala LP, Caprini JA, Lee CE, Arcelus JI, Reyna JJ, Cohen EB: A guide to venous thromboembolism risk factor assessment. J. Thromb. Thrombolysis 9(3), 253-262 (2000)

23. Ozaki H, Yasuda T, Ogasawara R, Sakamaki-Sunaga M, Naito H, Abe T: Effects of high-intensity and blood flow-restricted low-intensity resistance training on carotid arterial compliance: role of blood pressure during training sessions. Eur. J. Appl. Physiol. 113(1), 167-174 (2013)

24. Ozawa Y, Koto T, Shinoda H, Tsubota K: Vision loss by central retinal vein occlusion after Kaatsu training: a case report. Medicine (Baltimore) 94, e1515 (2015)

25. Rossow LM, Fahs CA, Loenneke JP, Thiebaud RS, Sherk VD, Abe T, Bemben MG: Cardiovascular and perceptual responses to blood-flow-restricted resistance exercise with differing restrictive cuffs. Clin. Physiol. Funct. Imaging 32(5), 331-337 (2012)

26. Sakamaki M, Yasuda T, Abe T: Comparison of low-intensity blood flow-restricted training-induced muscular hypertrophy in eumenorrheic women in the follicular phase and luteal phase and age-matched men. Clin. Physiol. Funct. Imaging 32(3), 185-191 (2012)

27. Spranger MD, Krishnan AC, Levy PD, O’Leary DS, Smith SA: Blood flow restriction training and the exercise pressor reflex: a call for concern. Am. J. Physiol. Heart Circ. Physiol. 309(9), H1440-H1452 (2015)

28. Yamanaka T, Farley RS, Caputo JL: Occlusion training increases muscular strength in division IA football players. J. Strength Cond. Res. 26(9), 2523-2529 (2012)

29. Yasuda T, Brechue WF, Fujita T, Shirakawa J, Sato Y, Abe T: Muscle activation during low-intensity muscle contractions with restricted blood flow. J. Sports Sci. 27(5), 479-489 (2009)

30. Yasuda T, Fujita S, Ogasawara R, Sato Y, Abe T: Effects of low-intensity bench press training with restricted arm muscle blood flow on chest muscle hypertrophy: a pilot study. Clin. Physiol. Funct. Imaging 30(5), 338-343 (2010) 
31. Yasuda T, Ogasawara R, Sakamaki M, Bemben MG, Abe T: Relationship between limb and trunk muscle hypertrophy following high-intensity resistance training and blood flow-restricted low-intensity resistance training. Clin. Physiol. Funct. Imaging 31(5), 347-351 (2011)

32. Yasuda T, Ogasawara R, Sakamaki M, Ozaki H, Sato Y, Abe T: Combined effects of low-intensity blood flow restriction training and high-intensity resistance training on muscle strength and size. Eur. J. Appl. Physiol. 111, $2525-2533(2011)$ 